Pacific

Journal of

Mathematics

NOVIKOV-TYPE INEQUALITIES FOR VECTOR FIELDS WITH NON-ISOLATED ZERO POINTS

Huitao Feng and Enli Guo

Volume $201 \quad$ No. 1

November 2001 


\title{
NOVIKOV-TYPE INEQUALITIES FOR VECTOR FIELDS WITH NON-ISOLATED ZERO POINTS
}

\author{
Huitao Feng and Enli Guo
}

\begin{abstract}
In this paper we prove some Novikov-type inequalities for vector fields with non-isolated zero points, which generalize some results of Shubin, 1996. As a consequence, we obtain an analytic proof of Hopf index theorem for vector fields which are nondegenerate in the sense of Bott.
\end{abstract}

\section{Introduction.}

In the article [5], Shubin presented a detailed treatment of the Novikov inequalities for vector fields with isolated zero points, to which Novikov sketched a proof in the appendix to [4]. As a consequence, a direct analytic proof of the Hopf index theorem for these vector fields is given. On the other hand, Braverman and Farber [2] obtained some Novikov-type inequalities for closed 1-forms with non-isolated zero points. In [3], we extended some results of Shubin [5] to a transversal section of a general oriented real vector bundle with the same dimension as its base manifold by constructing a super-twisted Dirac operator.

In this paper, we study the case of vector fields with non-isolated zero points. More precisely, let $X$ be a closed, oriented and connected Riemannian manifold of dimension $n$ and let $v$ be a vector field on $X$. Set

$$
Y=\{y \in X \mid v(y)=0\} .
$$

Then $Y$ can be expressed as a finite disjoint union of closed and connected subsets $Y_{k}, k=1,2, \ldots, m$. In this paper we assume that $v$ satisfies the following conditions for each $k=1,2, \ldots, m$ :

(C.1) $Y_{k}$ is a submanifold of $X$ of dimension $l_{k}$. In this case, the Lie derivative

$$
\mathcal{L}_{v}:\left.\left.T X\right|_{Y_{k}} \rightarrow T X\right|_{Y_{k}}
$$

is a homomorphism with kernel $T Y_{k}$.

$$
L_{v, k}=P^{N_{k}} \mathcal{L}_{v} P^{N_{k}}: N_{k} \rightarrow N_{k}
$$

is an isomorphism, where $N_{k}$ is the normal bundle of $T Y_{k}$ in $\left.T X\right|_{Y_{k}}$ with respect to the induced Euclidean inner product on $\left.T X\right|_{Y}$ from that on $T X$ and $P^{N_{k}}$ denotes the orthogonal projection from $\left.T X\right|_{Y_{k}}$ to $N_{k}$. 
(C.3) For any $Z \in N_{k}$,

$$
P^{T Y_{k}} \mathcal{L}_{v}(Z)=0
$$

where $P^{T Y_{k}}$ denotes the orthogonal projection from $\left.T X\right|_{Y_{k}}$ to $T Y_{k}$.

In this paper, by using Witten's deformation idea (cf. [7]) and BismutLebeau's technique (cf. [1]), we establish certain Novikov-type inequalities for vector fields verifying conditions (C.1)-(C.3) (Theorem 4.1). As a consequence, we obtain an analytic proof of Hopf index theorem (Theorem 4.2) for vector fields which are nondegenerate in the sense of Bott (in this case the condition (C.3) need not be used). Our result may be seen as a generalization of Shubin [5] and an analogue of Braverman and Farber [2] in the case of vector fields. The key step in our approach is that on each $Y_{k}$ we can define an index ind $\left(v, Y_{k}\right)$ and a line bundle $o_{Y_{k}}(v)$ by using the result of Shubin [5]. This line bundle has also played a role in the paper of Zhang [8] on his counting formula for the real Kervaire semi-characteristic.

\section{A deformed de Rham-Hodge operator $D_{T}^{X}$.}

In this section, we will define a deformed de Rham-Hodge operator $D_{T}^{X}$ by a vector field $v$ with the conditions (C.1)-(C.3) and discuss the local behavior of $D_{T}^{X}$ near the zero points set $Y$ of $v$ as $T \rightarrow \infty$. To do this, we need to study the geometry of the submanifold $Y$ and introduce some related differential operators. Especially, we will define a line bundle on $Y$ through the behavior of $v$ near $Y$, which is crucial to our problem.

Let $g^{T X}$ be the Riemannian metric on $X$ and $\nabla^{T X}$ be the associated Levi-Civita connection. We have the standard de Rham-Hodge operator

$$
D^{X}=d+\delta: \Gamma\left(\Lambda^{*}\left(T^{*} X\right)\right) \rightarrow \Gamma\left(\Lambda^{*}\left(T^{*} X\right)\right),
$$

which is a first order self-adjoint elliptic operator. Set

$$
D_{ \pm}^{X}: \Gamma\left(\Lambda^{\text {even/odd }}\left(T^{*} X\right)\right) \rightarrow \Gamma\left(\Lambda^{\text {odd } / \text { even }}\left(T^{*} X\right)\right) .
$$

We have

$$
\text { ind } D_{+}^{X}=\chi(X) \text {, }
$$

where $\chi(X)$ denotes the Euler characteristic of $X$.

For any vector field $v$, define in this paper

$$
c(v)=\varepsilon(v)-\iota(v), \quad \hat{c}(v)=\varepsilon(v)+\iota(v),
$$

where $\varepsilon$ and $\iota$ are the standard exterior and interior multiplications on $\Lambda^{*}\left(T^{*} X\right)$, respectively. Then for a vector field $v$ on $X$ with the conditions (C.1)-(C.3), we can define a deformed de Rham-Hodge operator

$$
D_{T}^{X}=D^{X}+T \hat{c}(v): \Gamma\left(\Lambda^{*}\left(T^{*} X\right)\right) \rightarrow \Gamma\left(\Lambda^{*}\left(T^{*} X\right)\right) .
$$

Set

$$
D_{T, \pm}^{X}=D_{ \pm}^{X}+T \hat{c}(v): \Gamma\left(\Lambda^{\text {even/odd }}\left(T^{*} X\right)\right) \rightarrow \Gamma\left(\Lambda^{\text {odd/even }}\left(T^{*} X\right)\right)
$$




$$
b_{ \pm}(v, T)=\operatorname{ker} \operatorname{dim} D_{T, \pm}^{X} .
$$

We have

$$
b_{+}(v, T)-b_{-}(v, T)=\chi(X) .
$$

Lemma 2.1. For any open neighborhood $\mathcal{U}$ of $Y$, there exist constants $a>$ $0, b>0$ and $T_{0}>0$ such that for any $s \in \Gamma\left(\Lambda^{*}\left(T^{*} X\right)\right)$ with $\operatorname{Supp} s \subset X \backslash \mathcal{U}$ and any $T \geq T_{0}$, we have the following estimate for Sobolev norms,

$$
\left\|D_{T}^{X} s\right\|_{0}^{2} \geq a\left(\|s\|_{1}^{2}+(T-b)\|s\|_{0}^{2}\right) .
$$

Proof. Since $D_{T}^{X}$ is formally self-adjoint and

$$
\left(D_{T}^{X}\right)^{2}=\left(D^{X}\right)^{2}+T\left[D^{X}, \hat{c}(v)\right]+T^{2}|v|^{2},
$$

we have

$$
\left\|D_{T}^{X} s\right\|_{0}^{2}=\left\|D^{X} s\right\|_{0}^{2}+T\left\langle\left[D^{X}, \hat{c}(v)\right] s, s\right\rangle+T^{2}\left\langle|v|^{2} s, s\right\rangle .
$$

Since $\left[D^{X}, \hat{c}(v)\right]$ is a zero order operator, which can be verified easily, and $v \neq 0$ on $X \backslash \mathcal{U}$, there exist constants $\widetilde{a}>0, \widetilde{b}>0$, such that for $T>0$ we have

$$
\left\|D_{T}^{X} s\right\|_{0}^{2} \geq\left\|D^{X} s\right\|_{0}^{2}+\widetilde{a} T^{2}\|s\|_{0}^{2}-\widetilde{b} T\|s\|_{0}^{2} .
$$

By Garding's inequality, there exist constants $a>0, \widetilde{c}>0$ such that

$$
\left\|D^{X} s\right\|_{0}^{2} \geq a\|s\|_{1}^{2}-\widetilde{c}\|s\|_{0}^{2} .
$$

Hence

$$
\begin{aligned}
\left\|D_{T}^{X} s\right\|_{0}^{2} & \geq a\|s\|_{1}^{2}+\left(\widetilde{a} T^{2}-\widetilde{b} T-\widetilde{c}\right)\|s\|_{0}^{2} \\
& \geq a\left(\|s\|_{1}^{2}+(T-b)\|s\|_{0}^{2}\right)
\end{aligned}
$$

for some constants $b>0, T_{0}>0$ and any $T \geq T_{0}$.

By Lemma 2.1, we can localize our problem to a sufficiently small neighborhood of $Y$. For simplicity, we always write $Y$ instead of $Y_{k}$ and assume $\operatorname{dim} Y=l$. We have the following orthogonal decomposition

$$
\left.T X\right|_{Y}=T Y \oplus N,
$$

where $N$ denotes the normal bundle of $T Y$ in $\left.T X\right|_{Y}$. Denote the projection $N \rightarrow Y$ by $\pi$ and the orthogonal projection $\left.T X\right|_{Y} \rightarrow T Y\left(\right.$ resp. $\left.T X\right|_{Y} \rightarrow N$ ) by $P^{T Y}$ (resp. $P^{N}$ ). Let $\nabla^{\left.T X\right|_{Y}}$ denote the restriction of $\nabla^{T X}$ to $\left.T X\right|_{Y}$. Set

$$
\nabla^{T Y}=P^{T Y} \nabla^{\left.T X\right|_{Y}} P^{T Y}, \quad \nabla^{N}=P^{N} \nabla^{\left.T X\right|_{Y}} P^{N}, \quad \nabla^{\left.T X\right|_{Y}, \oplus}=\nabla^{T Y} \oplus \nabla^{N} .
$$

Set

$$
\mathrm{A}=\nabla^{\left.T X\right|_{Y}}-\nabla^{\left.T X\right|_{Y}, \oplus,}
$$


which is the second fundamental form of $Y$. In this paper we always use the notation

$$
\left\{e_{1}, \ldots, e_{l}, f_{l+1}, \ldots, f_{n}\right\}
$$

to denote a local orthonormal frame for $\left.T X\right|_{Y}$ with $\left\{e_{1}, \ldots, e_{l}\right\}$ being an orthonormal frame for $T Y$ and $\left\{f_{l+1}, \ldots, f_{n}\right\}$ an orthonormal frame for $N$.

Following Shubin [5] and Zhang [8], we can define a line bundle on $Y$ through the vector field $v$. Set

$$
c_{\alpha, \beta}=\left\langle L_{v} f_{\alpha}, f_{\beta}\right\rangle, \quad C=\left\{c_{\alpha, \beta}\right\}, \quad|C|=\sqrt{C^{t} C} .
$$

Then

$$
\widehat{L}_{v}=\operatorname{tr}|C|+\sum_{\alpha, \beta=l+1}^{n} c_{\alpha, \beta} c\left(f_{\beta}\right) \hat{c}\left(f_{\alpha}\right): \Lambda^{*}\left(N^{*}\right) \rightarrow \Lambda^{*}\left(N^{*}\right),
$$

is a well-defined bundle homomorphism. Set

$$
\text { ind }(v, Y)=\operatorname{sign} \operatorname{det} C, \quad o_{Y}(v)=\operatorname{ker} \widehat{L}_{v} .
$$

Clearly, ind $(v, Y)$ and $o_{Y}(v)$ are also well-defined. The following lemma is an anologue of Proposition 2.21 in [5] and the (2.12), (2.13), (2.13)' in [8, Sect. 2.b].

Lemma 2.2. $o_{Y}(v)$ is a one dimensional subbundle of $\Lambda^{*}\left(N^{*}\right)$ over $Y$. Moreover,

$$
\begin{array}{ll}
o_{Y}(v) \subset \Lambda^{\text {even }}\left(N^{*}\right), & \text { if } \text { ind }(v, Y)=1, \\
o_{Y}(v) \subset \Lambda^{\text {odd }}\left(N^{*}\right), & \text { if } \text { ind }(v, Y)=-1 .
\end{array}
$$

Note that $\Lambda^{*}\left(T^{*} Y\right) \otimes o_{Y}(v)$ is a bundle twisted by an Euclidean flat line bundle $o_{Y}(v)$. Let $\nabla$ be the Euclidean connection on $o_{Y}(v)$ induced by the orthogonal projection from $\Lambda^{*}\left(N^{*}\right)$ to $o_{Y}(v)$. Clearly, for any local orthonormal section $\rho$ of $o_{Y}(v)$, we have $\nabla \rho=0$. We can define a twisted de Rham-Hodge operator by

$$
D^{Y}=\sum_{i=1}^{l} c\left(e_{i}\right) \widetilde{\nabla}_{e_{i}}^{Y}: \Gamma\left(\Lambda^{*}\left(T^{*} Y\right) \otimes o_{Y}(v)\right) \rightarrow \Gamma\left(\Lambda^{*}\left(T^{*} Y\right) \otimes o_{Y}(v)\right),
$$

where $\widetilde{\nabla}^{Y}=\nabla^{T Y} \otimes 1+1 \otimes \nabla$. Set

$$
D_{ \pm}^{Y}=D^{Y}: \Gamma\left(\Lambda^{\text {even/odd }}\left(T^{*} Y\right) \otimes o_{Y}(v)\right) \rightarrow \Gamma\left(\Lambda^{\text {odd/even }}\left(T^{*} Y\right) \otimes o_{Y}(v)\right) .
$$

Then $D_{-}^{Y}$ is the formal adjoint of $D_{+}^{Y}$. By Hodge theory, we have

$$
\text { ind } D_{+}^{Y}=\chi(Y)=\operatorname{dim} H^{\text {even }}\left(Y, o_{Y}(v)\right)-\operatorname{dim} H^{\text {odd }}\left(Y, o_{Y}(v)\right) \text {. }
$$

To have a good understanding about the local behavior of the operator $D_{T}^{X}$ near $Y$, we need study the geometry of $Y$ and the Taylor expansion of $v$ near $Y$. 
For $y \in Y$ and $Z \in N_{y}$, let

$$
t \in \mathbf{R} \rightarrow x_{t}=\exp _{y}^{X}(t Z) \in X,
$$

be the geodesic in $X$ such that $x_{0}=y, d x /\left.d t\right|_{t=0}=Z$. For $\epsilon>0$, set

$$
B_{\epsilon}=\{Z \in N|| Z \mid<\epsilon\} .
$$

Since $X$ and $Y$ are compact, there exists an $\epsilon_{0}>0$ such that for $0<\epsilon<\epsilon_{0}$, the map $(y, Z) \in N \rightarrow \exp { }_{y}^{X}(Z) \in X$ is a diffeomorphism from $B_{\epsilon}$ to a tubular neighborhood $\mathcal{U}_{\epsilon}$ of $Y$ in $X$. From now on, we will identify $B_{\epsilon}$ with $\mathcal{U}_{\epsilon}$ and use the notation $x=(y, Z)$ instead of $x=\exp _{y}^{X}(Z)$.

Let $d \sigma_{X}, d \sigma_{Y}$ and $d \sigma_{N_{y}}$ be the volume elements of $X, Y$, and fiber $N_{y}$ at $y$, respectively. Let $k(y, Z)$ be the smooth positive function defined on $B_{\epsilon_{0}}$ by the equation

$$
d \sigma_{X}(y, Z)=k(y, Z) d \sigma_{Y}(y) d \sigma_{N_{y}}(Z) .
$$

The function $k$ has a positive lower bound on $\mathcal{U}_{\frac{\epsilon_{0}}{2}}$. Clearly if $y \in Y$, then $k(y)=1$. Let $d^{N}$ denote the exterior differential along the fibres of $N$. Then for any $y \in Y, Z \in N_{y}$, we have the following formula from [1, Sect. $8,(8.25)]$

$$
d^{N} k(Z)=-\left\langle\sum_{i=1}^{l} \mathrm{~A}\left(e_{i}\right) e_{i}, Z\right\rangle .
$$

Let $\mathbf{E}$ (resp. E) be the set of smooth sections of $\pi^{*} \Lambda^{*}\left(\left.T^{*} X\right|_{Y}\right.$ ) (resp. $\Lambda^{*}\left(T^{*} X\right)$ ). If $s_{1}, s_{2} \in \mathbf{E}$ have compact supports, we define (cf. [1, Definition $8.15])$

$$
\left\langle s_{1}, s_{2}\right\rangle=\int_{Y}\left(\int_{N_{y}}\left\langle s_{1}, s_{2}\right\rangle(y, Z) d \sigma_{N_{y}}(Z)\right) d \sigma_{Y}(y) .
$$

By the trivialization of $\Lambda^{*}\left(T^{*} X\right)$ on $\mathcal{U}_{\epsilon}$ along the geodesics (16), if $s \in$ $\mathbf{E}$ has compact support in $B_{\epsilon_{0}}$, we can consider $s$ as a smooth section of $\Lambda^{*}\left(T^{*} X\right)$ with compact support in $B_{\epsilon_{0}}$. Let $\mathrm{E}_{\epsilon}$ (resp. $\mathbf{E}_{\epsilon}$ ) be the set of smooth sections of $\Lambda^{*}\left(T^{*} X\right)$ (resp. $\pi^{*}\left(\Lambda^{*}\left(\left.T^{*} X\right|_{Y}\right)\right)$ ) with compact support in $B_{\epsilon}$. One verifies easily that $k^{\frac{1}{2}} D^{X} k^{\frac{-1}{2}}$ acts as a formal self-adjoint operator on $\mathbf{E}_{\epsilon}$ with respect to the inner product (20).

Using the connection $\nabla^{N}$, we can split the tangent bundle $T N$ as

$$
T N=T^{H} \oplus T^{V} N,
$$

where $T^{H} N$ is the horizontal vector bundle and $T^{V} N$ is the vertical vector bundle which can be identified with $N$ naturally. Let $e_{i}^{H}$ denote the 
horizontal lifting of $e_{i}$. By the frame (10) we define

$$
\begin{gathered}
D^{H}=\sum_{i=1}^{l} c\left(e_{i}\right) \pi^{*} \nabla_{e_{i}^{H}}^{\left.T X\right|_{Y}, \oplus}: \mathbf{E} \rightarrow \mathbf{E}, \\
D^{N}=\sum_{\alpha=l+1}^{n} c\left(f_{\alpha}\right) \pi^{*} \nabla_{f_{\alpha}}^{\left.T X\right|_{Y}, \oplus}: \mathbf{E} \rightarrow \mathbf{E} .
\end{gathered}
$$

Clearly, the definitions of $D^{H}, D^{N}$ are independent of the choice of the frames (10), so $D^{H}, D^{N}$ are two well-defined first order differential operators acting on $\mathbf{E}$. Moreover, the operator $D^{N}$ acts along the fibres $N_{y}$, and that $D^{H}, D^{N}$ and $D^{H}+D^{N}$ are self-adjoint with respect to the $L^{2}$ inner product (20). Particularly, the operator $D^{H}+D^{N}$ is also a self-adjoint elliptic operator. (cf. [1, Sect. 8.h]).

By the parallel transport of the frame (10) along the geodesics (16), we get a local orthonormal frame for $\left.T X\right|_{\mathcal{U}_{\epsilon}}$

$$
\left\{e_{1}^{\tau}, \ldots, e_{l}^{\tau}, f_{l+1}^{\tau}, \ldots, f_{n}^{\tau}\right\} .
$$

Let $\left(z_{l+1}, \ldots, z_{n}\right)$ be the coordinate on the fibre $N_{y}$ with respect to $\left\{f_{l+1}\right.$, $\left.\ldots, f_{n}\right\}$. Then the vector field $v$ has an expression near $Y$ :

$$
v=\sum_{i=1}^{l} v_{i} e_{i}^{\tau}+\sum_{\alpha=l+1}^{n} v_{\alpha} f_{\alpha}^{\tau} .
$$

Set

$$
v_{Y}=\sum_{i=1}^{l} v_{i} e_{i}^{\tau}, \quad v_{N}=\sum_{\alpha=l+1}^{n} v_{\alpha} f_{\alpha}^{\tau} .
$$

Clearly, the definitions of $v_{Y}$ and $v_{N}$ are independent of the choice of the frame (10). Set

$$
\begin{gathered}
v_{Y, 1}(y, Z)=\sum_{i=1}^{l} \sum_{\alpha=l+1}^{n} \frac{\partial v_{i}}{\partial z_{\alpha}}(y) z_{\alpha} e_{i}^{\tau}(y, Z), \\
v_{Y, 2}(y, Z)=\frac{1}{2} \sum_{i=1}^{l} \sum_{\alpha, \beta=l+1}^{n} \frac{\partial^{2} v_{i}}{\partial z_{\alpha} \partial z_{\beta}}(y) z_{\alpha} z_{\beta} e_{i}^{\tau}(y, Z), \\
v_{N, 1}(y, Z)=\sum_{\alpha=l+1}^{n} \sum_{\beta=l+1}^{n} \frac{\partial v_{\alpha}}{\partial z_{\beta}}(y) z_{\beta} f_{\alpha}^{\tau}(y, Z), \\
v_{N, 2}(y, Z)=\frac{1}{2} \sum_{\alpha=l+1}^{n} \sum_{\beta, \gamma=l+1}^{n} \frac{\partial^{2} v_{\alpha}}{\partial z_{\beta} \partial z_{\gamma}}(y) z_{\beta} z_{\gamma} f_{\alpha}^{\tau}(y, Z) .
\end{gathered}
$$


One also verifies easily that the definitions of $v_{Y, 1}, v_{Y, 2}, v_{N, 1}, v_{N, 2}$ are independent of the choices of the frames (10). By the condition (C.3), we have $v_{Y, 1}=0$ easily and

$$
v(y, Z)=v_{Y, 2}+v_{N, 1}+v_{N, 2}+\mathrm{O}\left(|Z|^{3}\right) .
$$

By the definition (11) of $c_{\alpha, \beta}$, we have

$$
v_{N, 1}(y, Z)=\sum_{\alpha=l+1}^{n} \sum_{\beta=l+1}^{n} c_{\alpha, \beta}(y) z_{\beta} f_{\alpha}^{\tau}(y, Z) .
$$

We can also define a vector field $\bar{v}_{Y}$ on $Y$ by

$$
\bar{v}_{Y}=\frac{1}{4} \nabla_{f_{\alpha}}^{\left.T X\right|_{Y}} C^{-t} C^{-1}\left(\nabla_{f_{\alpha}^{\tau}}^{T X}\right)^{t} v_{Y},
$$

where

$$
\nabla_{f_{\alpha}}^{\left.T X\right|_{Y}}=\left(\nabla_{f_{l+1}}^{\left.T X\right|_{Y}}, \ldots, \nabla_{f_{n}}^{\left.T X\right|_{Y}}\right)
$$

and $\left(\nabla_{f_{\alpha}^{\tau}}^{T X}\right)^{t}$ is the transpose of the formal matrix $\left(\nabla_{f_{l+1}^{\tau}}^{T X}, \ldots, \nabla_{f_{n}^{\tau}}^{T X}\right)$. One sees easily that $\bar{v}_{Y}$ is independent of the choice of frames (10). Therefore, the vector field $\bar{v}_{Y} \in \Gamma(T Y)$ is well-defined. Let

$$
s_{l+1}(y), s_{l+2}(y), \ldots, s_{n}(y)
$$

be the eigenvalues of $|C(y)|$. For a suitable orthonormal frame of $N$, we have

$$
|C(y)|=\operatorname{diag}\left(s_{l+1}, \ldots, s_{n}\right), \quad \text { with } \quad s_{i}>0 .
$$

Then the vector field $\bar{v}_{Y}$ can be expressed locally as

$$
\bar{v}_{Y}=\sum_{i=1}^{l} \sum_{\alpha=l+1}^{n} \frac{1}{s_{\alpha}^{2}} \frac{\partial^{2} v_{i}}{\partial z_{\alpha}^{2}} e_{i} .
$$

Now we can define some deformed operators as following

$$
\begin{gathered}
D_{v}^{Y}=D^{Y}+\hat{c}\left(\bar{v}_{Y}\right): \Gamma\left(\Lambda^{*}\left(T^{*} Y\right) \otimes o_{Y}(v)\right) \rightarrow \Gamma\left(\Lambda^{*}\left(T^{*} Y\right) \otimes o_{Y}(v)\right), \\
D_{T}^{H}=D^{H}+T \hat{c}\left(v_{Y, 2}\right), \\
D_{T}^{N}=D^{N}+T \hat{c}\left(v_{N, 1}\right) .
\end{gathered}
$$

Set

$$
\begin{aligned}
D_{v, \pm}^{Y}=D_{ \pm}^{Y}+\hat{c}\left(\bar{v}_{Y}\right): \Gamma\left(\Lambda^{\text {even/odd }}\left(T^{*} Y\right) \otimes o_{Y}(v)\right) \\
\rightarrow \Gamma\left(\Lambda^{\text {odd/even }}\left(T^{*} Y\right) \otimes o_{Y}(v)\right)
\end{aligned}
$$

Then we have $\chi(Y)=b_{+}(v, Y)-b_{-}(v, Y)$. 
Note that $D_{T}^{N}$ is actually a deformed de Rham-Hodge operator acting fibre-wisely on $\Gamma\left(\pi^{*} \Lambda^{*}\left(N^{*}\right)\right)$. An easy computation shows that

$$
\left(D_{T}^{N}\right)^{2}=-\sum_{\alpha=l+1}^{n}\left(\pi \nabla_{f_{\alpha}}^{\left.T X\right|_{Y}, \oplus}\right)^{2}+T^{2}\langle|C| Z,|C| Z\rangle-T \operatorname{tr}|C|+T \widehat{L}_{v}
$$

By the spectral theory of harmonic oscillators, we get the following lemma which is an analogue of Corollary 2.22 of Shubin [5] and Lemma 2.3 of Zhang $[8]$ :

Lemma 2.3. Take $T>0$. Then for any $y \in Y$, the operator $\left(D_{T}^{N}\right)^{2}$ acting on $\Gamma\left(\Lambda^{*}\left(N_{y}^{*}\right)\right)$ over $N_{y}$ is nonnegative with kernel being one dimensional and generated by

$$
\exp \left(-\frac{T}{2}\langle|C| Z,|C| Z\rangle\right) \rho(y)
$$

where $\rho(y)$ is a generator of ker $\widehat{L}_{v}(y)$ with unit length, $\rho(y) \in \Lambda^{\text {even }}\left(N_{y}^{*}\right)$ if $\operatorname{det} C(y)>0$, and $\rho(y) \in \Lambda^{\text {odd }}\left(N_{y}^{*}\right)$ if $\operatorname{det} C(y)<0$. Furthermore, the nonzero eigenvalues of $\left(D_{T}^{N}\right)^{2}$ are all $\geq T A$ for some positive constant $A$ which can be chosen to be independent of $y$.

Now similar to Theorem 8.18 in [1], we can give the following lemma which describes the local behavior of $D_{T}^{X}$ as $T \rightarrow \infty$.

Lemma 2.4. As $T \rightarrow \infty$, we have the following asymotopic formula on $\mathbf{E}_{\epsilon}$ :

$$
k^{1 / 2} D_{T}^{X} k^{-1 / 2}=D_{T}^{H}+D_{T}^{N}+T \hat{c}\left(v_{N, 2}\right)+Q+R_{T},
$$

where

$$
\begin{gathered}
Q=-\frac{1}{2} \sum_{i, j=1}^{l} \sum_{\alpha=l+1}^{n}\left\langle\mathrm{~A}\left(e_{i}\right) e_{j}, f_{\alpha}\right\rangle c\left(e_{i}\right) \hat{c}\left(e_{j}\right) \hat{c}\left(f_{\alpha}\right), \\
R_{T}=O\left(|Z| \partial^{H}+|Z|^{2} \partial^{N}+|Z|+T\left|Z^{3}\right|\right)
\end{gathered}
$$

and $\partial^{H}, \partial^{N}$ represent horizontal and vertical differential operators, respectively.

Proof. Note that near $Y$ we have

$$
D_{T}^{X}=\sum_{i=1}^{l} c\left(e_{i}\right) \nabla_{e_{i}^{\tau}}^{T X}+\sum_{\alpha=l+1}^{n} c\left(f_{\alpha}\right) \nabla_{f_{\alpha}^{\tau}}^{T X}+T \hat{c}(v) .
$$


Similar to the proof of Theorem 8.18 in $[\mathbf{1}]$, we have

$$
\begin{aligned}
k^{1 / 2} D_{T}^{X} k^{-1 / 2}= & D_{T}^{H}+D_{T}^{N}+T \hat{c}\left(v_{N, 2}\right)-\frac{1}{2} \sum_{\alpha=l+1}^{n} d^{N} k\left(f_{\alpha}\right) c\left(f_{\alpha}\right) \\
& +\sum_{i=1}^{l} c\left(e_{i}\right) \pi^{*} \mathrm{~A}\left(e_{i}^{H}\right)+R_{T} .
\end{aligned}
$$

By an easy computation and (19), we have

$$
\sum_{i=1}^{l} c\left(e_{i}\right) \pi^{*} \mathrm{~A}\left(e_{i}^{H}\right)=\frac{1}{2} \sum_{\alpha=l+1}^{n} d^{N} k\left(f_{\alpha}\right) c\left(f_{\alpha}\right)+Q .
$$

Hence we have

$$
k^{1 / 2} D_{T}^{X} k^{-1 / 2}=D_{T}^{H}+D_{T}^{N}+T \hat{c}\left(v_{N, 2}\right)+Q+R_{T} .
$$

\section{Various estimates on the $D_{T, j}$ 's as $T \rightarrow \infty$.}

In this section, we will give a suitable decomposition of $D_{T}^{X}$ as $\sum_{j=1}^{4} D_{T, j}$ and establish some estimates about $D_{T, j}$ as $T \rightarrow \infty$ by using Bismut-Lebeau's techniques ([1, Sect. 9]).

For any $\mu \geq 0$, let $\mathrm{E}^{\mu}$ (resp. $\mathbf{E}^{\mu}$, resp. $\mathrm{F}^{\mu}$ ) be the set of sections of $\Lambda^{*}\left(T^{*} X\right)$ on $X$ (resp. of $\pi^{*} \Lambda^{*}\left(\left.T^{*} X\right|_{Y}\right)$ on the total space of $N$, resp. of $\Lambda^{*}\left(T^{*} Y\right) \otimes o_{Y}(v)$ on $\left.Y\right)$ which lie in the $\mu$-th Sobolev spaces. Let \|\|$_{\mathrm{E}^{\mu}}$ (resp. \|\|$_{\mathbf{E}^{\mu}}$, resp. \|\|$\left._{\mathrm{F}^{\mu}}\right)$ be the Sobolev norm on $\mathrm{E}^{\mu}$ (resp. $\mathbf{E}^{\mu}$, resp. $\mathrm{F}^{\mu}$ )

Let $\gamma: \mathbf{R} \rightarrow[0,1]$ be a smooth even function with $\gamma(a)=1$ if $|a| \leq \frac{1}{2}$ and $\gamma(a)=0$ if $|a| \geq 1$. Set

$$
\gamma_{\epsilon}(y, Z)=\gamma\left(\frac{|Z|}{\epsilon}\right)
$$

for any $y \in Y, Z \in N_{y}$ and $\epsilon \in\left(0, \epsilon_{0}\right)$, where $\epsilon_{0}$ is chosen as in Section 2 . When there is no confusion, we denote it by $\gamma_{\epsilon}(Z)$.

For any $T>0$ and $y \in Y$, set

$$
\begin{gathered}
\alpha_{T}(y)=\int_{N_{y}} \gamma_{\epsilon}^{2}(Z)|\operatorname{det} C(y)| \exp (-T\langle|C(y)| Z,|C(y)| Z\rangle) d \sigma_{N_{y}}(Z), \\
G_{T}(y, Z)=\alpha_{T}^{-\frac{1}{2}}(y) \gamma_{\epsilon}(Z) \sqrt{\operatorname{det}|C(y)|} \exp \left(-\frac{T}{2}\langle|C(y)| Z,|C(y)| Z\rangle\right) .
\end{gathered}
$$

For $\mu \geq 0, T>0$, define linear maps $I_{T}: \mathrm{F}^{\mu} \rightarrow \mathbf{E}^{\mu}$ and $J_{T}: \mathrm{F}^{\mu} \rightarrow \mathrm{E}^{\mu}$ by

$$
I_{T} u=G_{T} \pi^{*} u, \quad J_{T} u=k^{-1 / 2} I_{T} u
$$

for any $u \in \mathrm{F}^{\mu}$. It is easy to see that $I_{T}, J_{T}$ are isometries from $\mathrm{F}^{0}$ onto their images. For $\mu \geq 0, T>0$, let $\mathbf{E}_{T}^{\mu}\left(\operatorname{resp} . \mathrm{E}^{\mu}\right)$ be the image of $\mathrm{F}^{\mu}$ in $\mathbf{E}^{\mu}$ 
(resp. $\mathrm{E}^{\mu}$ ) under $I_{T}$ (resp. $J_{T}$ ) and let $\mathbf{E}_{T}^{0, \perp}$ (resp. $\mathrm{E}_{T}^{0, \perp}$ ) be the orthogonal complement of $\mathbf{E}_{T}^{0}\left(\operatorname{resp} . \mathrm{E}_{T}^{0}\right)$ in $\mathbf{E}^{0}\left(\operatorname{resp} . \mathrm{E}^{0}\right)$ and let $p_{T}, p_{T}^{\perp}\left(\operatorname{resp} . \bar{p}_{T}, \bar{p}_{T}^{\perp}\right)$ be the orthogonal projection operators from $\mathbf{E}^{0}$ (resp. $\mathrm{E}^{0}$ ) onto $\mathbf{E}_{T}^{0}, \mathbf{E}_{T}^{0, \perp}$ (resp. $\mathrm{E}_{T}^{0}, \mathrm{E}_{T}^{0, \perp}$ ), respectively. Set

$$
\mathrm{E}^{\mu, \perp}=\mathrm{E}^{\mu} \cap \mathrm{E}_{T}^{0, \perp} .
$$

Then $\mathrm{E}^{0}$ splits orthogonally into

$$
\mathrm{E}^{0}=\mathrm{E}_{T}^{0} \oplus \mathrm{E}_{T}^{0, \perp}
$$

Since the map $s \in \mathbf{E}^{0} \rightarrow k^{-1 / 2} s \in \mathrm{E}^{0}$ is an isometry, we see that the map $s \rightarrow k^{-1 / 2} s$ identifies the Hilbert space $\mathbf{E}_{T}^{0}$ and $\mathrm{E}_{T}^{0}$. According to the decomposition (48) we set:

$$
D_{T, 1}=\bar{p}_{T} D_{T}^{X} \bar{p}_{T}, \quad D_{T, 2}=\bar{p}_{T} D_{T}^{X} \bar{p}_{T}^{\perp}, \quad D_{T, 3}=\bar{p}_{T}^{\perp} D_{T}^{X} \bar{p}_{T}, \quad D_{T, 4}=\bar{p}_{T}^{\perp} D_{T}^{X} \bar{p}_{T}^{\perp} .
$$

Then

$$
D_{T}^{X}=D_{T, 1}+D_{T, 2}+D_{T, 3}+D_{T, 4} .
$$

Let $\mathbf{q}$ denote the orthogonal projection from $\pi^{*} \Lambda^{*}\left(\left.T^{*} X\right|_{Y}\right)$ on $\pi^{*}\left(\Lambda^{*}\left(T^{*} Y\right) \otimes o_{Y}(v)\right)$.

In the following we will estimate $D_{T, j}$. Similar to Theorem 9.8 in [1], we have the following lemma.

Lemma 3.1. The following formula holds on $\Gamma\left(\Lambda^{*}\left(T^{*} Y\right) \otimes o_{Y}(v)\right)$ as $T \rightarrow$ $+\infty$

$$
J_{T}^{-1} D_{T, 1} J_{T}=D_{v}^{Y}+O\left(\frac{1}{\sqrt{T}}\right),
$$

where $O\left(\frac{1}{\sqrt{T}}\right)$ is a first order differential operator with smooth coefficients dominated by $C / \sqrt{T}$.

Proof. For any $u \in \mathrm{F}=\Gamma\left(\Lambda^{*}\left(T^{*} Y\right) \otimes o_{Y}(v)\right)$, we have

$$
\begin{aligned}
J_{T}^{-1} D_{T, 1} J_{T} u & =I_{T}^{-1} p_{T} k^{1 / 2} D_{T}^{X} k^{-1 / 2} p_{T} I_{T} u \\
& =I_{T}^{-1} p_{T}\left(D_{T}^{H}+D_{T}^{N}+T \hat{c}\left(v_{N, 2}\right)+Q+R_{T}\right) G_{T}(y, Z) \pi^{*} u .
\end{aligned}
$$

The only different term here from the proof of Theorem 9.8 in [1] in computations is

$$
\begin{aligned}
& I_{T}^{-1} p_{T} D_{T}^{H}\left(G_{T}(y, Z) \pi^{*} u\right) \\
& =I_{T}^{-1} p_{T} D^{H} G_{T}(y, Z) \pi^{*} u+T I_{T}^{-1} p_{T} \hat{c}\left(v_{Y, 2}\right) G_{T}(y, Z) \pi^{*} u .
\end{aligned}
$$

Similar to [1, Theorem 9.8], we have

$$
I_{T}^{-1} p_{T} D^{H} G_{T}(y, Z) \pi^{*} u=D^{Y} u \text {. }
$$


By the definitions of $p_{T}, G_{T}(y, Z)$ and $\hat{c}\left(v_{Y, 2}\right)$, we have

$$
I_{T}^{-1} p_{T} \hat{c}(v) \pi^{*} u=\frac{1}{2} I_{T}^{-1} \sum_{i=1}^{l} G_{T}(y, Z) \pi^{*}\left(\hat{c}\left(e_{i}\right) u\right) I_{1},
$$

where

$$
\begin{aligned}
I_{1}= & \frac{1}{\alpha_{T}} \int_{N_{y}} \gamma_{\epsilon}^{2}\left(\left|Z^{\prime}\right|\right) \operatorname{det}|C(y)| \exp \left(-T\left\langle|C(y)| Z^{\prime},|C(y)| Z^{\prime}\right\rangle\right) \\
& \sum_{\mu, \nu=l+1}^{n} \frac{\partial^{2} v_{i}}{\partial z_{\mu}^{\prime} \partial z_{\nu}^{\prime}}(y) z_{\mu}^{\prime} z_{\nu}^{\prime} d \sigma_{N_{y}}\left(Z^{\prime}\right) .
\end{aligned}
$$

By (31) and the symmetry of the integral $I_{1}$, we get

$$
\begin{gathered}
I_{1}=\frac{1}{\alpha_{T}} \prod_{\beta=l+1}^{n} s_{\beta} \int_{N_{y}} \gamma_{\epsilon}^{2}(|Z|) \exp \left(-T \sum_{\alpha=l+1}^{n} s_{\alpha}^{2} z_{\alpha}^{2}\right) \\
\cdot \sum_{\lambda=l+1}^{n} \frac{\partial^{2} v_{i}}{\partial z_{\lambda}^{2}}(y) z_{\lambda}^{2} d z_{l+1} \cdots d z_{n} .
\end{gathered}
$$

Set $\bar{z}_{\alpha}=s_{\alpha} z_{\alpha}$. We find that

$$
\begin{aligned}
I_{1}= & \frac{1}{2 T} \frac{1}{\alpha_{T}} \sum_{\lambda=l+1}^{n} \frac{1}{s_{\lambda}^{2}} \frac{\partial^{2} v_{i}}{\partial \bar{z}_{\lambda}^{2}}(y) \\
& \cdot\left(\int_{N_{y}} \gamma_{\epsilon}^{2} e^{-T|\bar{Z}|^{2}} d \sigma_{N_{y}}+\int_{N_{y}} e^{-T|\bar{Z}|^{2}} \frac{\partial \gamma_{\epsilon}^{2}}{\partial \bar{z}_{\lambda}} \bar{z}_{\lambda} d \sigma_{N_{y}}\right) .
\end{aligned}
$$

Note that

$$
\begin{gathered}
\frac{1}{\alpha_{T}} \int_{N_{y}} \gamma_{\epsilon}^{2} e^{-T|\bar{Z}|^{2}} d \sigma_{N_{y}}=1 \\
\frac{1}{2 T} \frac{1}{\alpha_{T}} \sum_{\lambda=l+1}^{n} \frac{1}{s_{\lambda}^{2}} \frac{\partial^{2} v_{i}}{\partial \bar{z}_{\lambda}^{2}}(y) \int_{N_{y}} \exp \left(-T|\bar{Z}|^{2}\right) \frac{\partial \gamma_{\epsilon}^{2}}{\partial \bar{z}_{\lambda}} \bar{z}_{\lambda} d \sigma_{N_{y}}=O\left(\frac{1}{T^{3 / 2}}\right)
\end{gathered}
$$

we have

$$
\begin{aligned}
& I_{T}^{-1} p_{T} \hat{c}\left(v_{Y, 2}\right)\left(G_{T}(y, Z) \pi^{*} u\right) \\
& =\frac{1}{4 T} \sum_{i=1}^{l} \hat{c}\left(\sum_{\lambda=l+1}^{n} \frac{1}{s_{\lambda}^{2}} \frac{\partial^{2} v_{i}}{\partial z_{\lambda}^{2}}(y) e_{i}\right) u+O\left(\frac{1}{T^{3 / 2}}\right) u .
\end{aligned}
$$

Combine (52) and (53) and by the definition (33) of $D_{v}^{Y}$, we have

$$
I_{T}^{-1} p_{T} \hat{c}\left(v_{Y, 2}\right)\left(G_{T}(y, Z) \pi^{*} u\right)=D_{v}^{Y} u+O\left(\frac{1}{\sqrt{T}}\right) u .
$$


Similar to the proofs of Theorem 9.10, Theorem 9.11 and Theorem 9.14 in [1, Sect. 9], we can prove the following lemma without any new difficulties.

Lemma 3.2. There exists $C_{1}>0, C_{2}>0$ and $T_{0}>0$ such that for any $T \geq T_{0}, s \in \mathrm{E}_{T}^{1, \perp}$ and $s^{\prime} \in \mathrm{E}_{T}^{1}$, we have

$$
\begin{gathered}
\left\|D_{T, 2} s\right\|_{\mathrm{E}^{0}} \leq C_{1}\left(\frac{\|s\|_{\mathrm{E}^{1}}}{\sqrt{T}}+\|s\|_{\mathrm{E}^{0}}\right) \\
\left\|D_{T, 3} s^{\prime}\right\|_{\mathrm{E}^{0}} \leq C_{1}\left(\frac{\left\|s^{\prime}\right\|_{\mathrm{E}^{1}}}{\sqrt{T}}+\left\|s^{\prime}\right\|_{\mathrm{E}^{0}}\right) \\
\left\|D_{T, 4} s\right\|_{\mathrm{E}^{0}} \geq C_{2}\left(\|s\|_{\mathrm{E}^{1}}+\sqrt{T}\|s\|_{\mathrm{E}^{0}}\right) .
\end{gathered}
$$

\section{The proofs of the main results.}

In this section, we will prove the main results in this paper by using the techniques of Bismut and Lebeau (cf. [1, Sect. 9.c)-f)]).

Denote the spectum of $D_{v}^{Y}$ by $\operatorname{Spec}\left(D_{v}^{Y}\right)$. Choose $c>0$ such that

$$
\operatorname{Spec}\left(D_{v}^{Y}\right) \cap[-2 c, 2 c]=\{0\} .
$$

Let $\mathrm{E}_{c}(T)$ denote the direct sum of the eigenspaces of $D_{T}^{X}$ with eigenvalues lying in $[-c, c]$. Then $\mathrm{E}_{c}(T)$ is a finite dimensional subspace of $\mathrm{E}^{0}$. Using the estimates for $D_{T, j}$ in Lemmas $3.1,3.2$ and proceeding as in ([1, pp. 117-125]) (also compare with Tian-Zhang [6, Sect. 4, Lemma 4.6, 4.7]), we have the following:

Proposition 4.1. There exists $T_{0}>0$ such that for any $T \geq T_{0}$, we have

$$
\operatorname{dim} \mathrm{E}_{c}(T)=\operatorname{dim} \operatorname{ker} D_{v}^{Y} .
$$

Set

$$
\begin{gathered}
\mathrm{E}_{c,+}(T)=\mathrm{E}_{c}(T) \cap \Gamma\left(\Lambda^{\text {even }}\left(T^{*} X\right)\right), \\
\mathrm{E}_{c,-}(T)=\mathrm{E}_{c}(T) \cap \Gamma\left(\Lambda^{\text {odd }}\left(T^{*} X\right)\right) .
\end{gathered}
$$

Then by the definition (37) of $b_{ \pm}\left(v, Y_{k}\right)$, we have

$$
\operatorname{dim} \mathrm{E}_{c, \pm}(T)=\sum_{\text {ind }\left(v, Y_{k}\right)=1} b_{ \pm}\left(v, Y_{k}\right)+\sum_{\text {ind }\left(v, Y_{k}\right)=-1} b_{\mp}\left(v, Y_{k}\right),
$$

for $c>0$ and $T \geq T_{0}>0$ in Proposition 4.1.

Now we get the following Novikov-type inequalities for vector fields $v$ with the conditions (C.1)-(C.3). 
Theorem 4.1 (Novikov-type inequalities). There exists $T_{0} \geq 0$ such that for any $T \geq T_{0}$, the following inequalities hold

$$
b_{ \pm}(v, T) \leq \sum_{\text {ind }\left(v, Y_{k}\right)=1} b_{ \pm}\left(v, Y_{k}\right)+\sum_{\text {ind }\left(v, Y_{k}\right)=-1} b_{\mp}\left(v, Y_{k}\right) .
$$

Proof. Since

$$
b_{ \pm}(v, T)=\operatorname{dim} \operatorname{ker} D_{T, \pm}^{X} \leq \operatorname{dim} \mathrm{E}_{c, \pm},
$$

we get (62) by (61).

Let $v \in \Gamma(T X)$ be a vector field which is nondegenerate in the sense of Bott. We can deform $v$ near its zero points set $Y$ such that $Y$, ind $(v, Y)$ and $o_{Y}(v)$ are unchanged under the deformation and the resulting $v$ satisfies the conditions (C.1)-(C.3). So we can prove the following Hopf index theorem analytically.

Theorem 4.2 (Hopf index theorem). Let $v$ be a nondegenerate vector field in the sense of Bott. Then we have

$$
\chi(X)=\sum_{k=1}^{m} \text { ind }\left(v, Y_{k}\right) \chi\left(Y_{k}\right) .
$$

Proof. We have

$$
\begin{aligned}
\chi(X)= & \operatorname{ind} D_{T,+}^{X} \\
= & \operatorname{ind}\left\{D_{T}^{X}: \mathrm{E}_{c,+}(T) \rightarrow \mathrm{E}_{c,-}(T)\right\} \\
= & \operatorname{dim} \mathrm{E}_{c,+}(T)-\operatorname{dim} \mathrm{E}_{c,-}(T) \\
= & \sum_{\operatorname{ind}\left(v, Y_{k}\right)=1}\left(b_{+}\left(v, Y_{k}\right)-b_{-}\left(v, Y_{k}\right)\right) \\
& +\sum_{\operatorname{ind}\left(v, Y_{k}\right)=-1}\left(b_{-}\left(v, Y_{k}\right)-b_{+}\left(v, Y_{k}\right)\right) \\
= & \sum_{k=1}^{m} \operatorname{ind}\left(v, Y_{k}\right) \chi\left(Y_{k}\right) .
\end{aligned}
$$

Remark 4.1. In the case of $v_{Y, 2}=0$, the Novikov-type inequalities (62) take the forms:

$$
\begin{aligned}
b_{ \pm}(v, T) \leq & \sum_{\text {ind }\left(v, Y_{k}\right)=1} \operatorname{dim} H^{\text {even } / \text { odd }}\left(Y_{k}, o_{Y_{k}}(v)\right) \\
& +\sum_{\text {ind }\left(v, Y_{k}\right)=-1} \operatorname{dim} H^{\text {odd } / \text { even }}\left(Y_{k}, o_{Y_{k}}(v)\right) .
\end{aligned}
$$


Acknowledgements. The authors would like to thank Professor Weiping Zhang for very helpful suggestions.

\section{References}

[1] J.-M. Bismut and G. Lebeau, Complex immersions and Quillen metrics, Publ. Math. IHES., 74 (1991), 80-125, MR 94a:58205, Zbl 784.32010.

[2] M. Braverman and M. Farber, Novikov type inequalities for differential forms with non-isolated zeros, Math. Proc. Camb. Phil. Soc., 122 (1997), 357-375, MR 99b:58220, Zbl 894.58012.

[3] H. Feng and E. Guo, A super-twisted Dirac operator and Novikov inequalities, Science in China (series A), 5 (2000), 470-480, CMP 1775757.

[4] S.P. Novikov and M.A. Shubin, Morse inequalities and von Neumann $\mathrm{II}_{2}$-factors, Soviet Math. Dokl., 34(1) (1987), 79-82, MR 88c:58065, Zbl 647.46049.

[5] M. Shubin, Novikov inequalities for vector fields, The Gelfand Mathematical Seminar, 1993-1995, Birkhäuser, Boston, (1996), 243-274, MR 97h:58150, Zbl 868.58006.

[6] Y. Tian and W. Zhang, Quantization formula for symplectic manifolds with boundary, Geom. Funct. Anal., 9 (1999), 596-640, CMP 1708436.

[7] E. Witten, Supersymmetry and Morse theory, J. Diff. Geom., 17 (1982), 661-692, MR 84b:58111, Zbl 499.53056.

[8] W. Zhang, A counting formula for the Kervaire semi-characteristic, Topology, 39 (2000), 643-655, MR 2000m:57040.

Received December 12, 1999 and revised May 3, 2000. This work was partially supported by the NNSF of China and the Qiu Shi Foundation.

NANKai Institute of Mathematics

NANKAI UNIVERSITY

Tianjin 300071

People's Republic of China

E-mail address: htfeng@nankai.edu.cn

The College of Applied Mathematics and Physics

Beijing Polytechnic University

BeiJing, 100000

People's Republic of China 